\title{
Enfoque por competencias y resultados escolares. Estudio longitudinal en el Principado de Asturias ${ }^{1}$
}

\section{Competency-based learning and school outcomes. A longitudinal study in the Principality of Asturias}

\author{
José Antonio Álvarez Castrillón² \\ jcastrillon@uniovi.es \\ Alejandro Rodríguez-Martín \\ rodriguezmalejandro@uniovi.es \\ Emilio Álvarez-Arregui \\ alvarezemilio@uniovi.es \\ Universidad de Oviedo, España
}

\section{Resumen:}

Las recientes leyes que han regulado el sistema educativo en España han coincidido en dos aspectos: la incorporación de las Competencias Básicas y la implantación de evaluaciones de diagnóstico para conocer su grado de desarrollo. La aplicación de estas pruebas en el Principado de Asturias desde el curso 2005-2006 convierte a esta región en pionera en su diseño y desarrollo. En este artículo presentamos un meta-análisis de los resultados obtenidos por el alumnado de $4^{\circ}$ de Edu-

\begin{abstract}
:
The recent laws regulating the education system in Spain have coincided in two main aspects: the introduction of the competency-based approach and the implementation of diagnostic evaluations to ascertain their degree of development. The application of these evaluations in the Principality of Asturias from the academic year 2005-2006 has enabled the region to be one of the pioneers in designing and carrying them out. This article presents a meta-analysis study of the results obtained
\end{abstract}

1 Este estudio ha sido posible gracias al apoyo, la documentación e información proporcionadas por el Servicio de Ordenación y Evaluación Educativa, perteneciente a la Dirección General de Formación Profesional, Desarrollo Curricular e Innovación Educativa de la Consejería de Educación, Cultura y Deporte del Principado de Asturias.

2 Dirección para correspondencia (correspondence address):

José Antonio Álvarez Castrillón. Departamento de Ciencias de la Educación. Facultad de Formación del Profesorado y Educación. Universidad de Oviedo. C/ Aniceto Sela s/n, Campus de Llamaquique. 33005 Oviedo (España). 
Enfoque por competencias y resultados escolares. Estudio longitudinal en el Principado de Asturias

José Antonio Álvarez Castrillón, Alejandro Rodríguez-Martín y Emilio Álvarez-Arregui

cación Primaria y $2^{\circ}$ de E.S.O. en las evaluaciones de diagnóstico durante los últimos seis cursos académicos (2006-2012), con el objetivo de conocer el impacto que el enfoque por competencias ha tenido en los resultados escolares de esta región. La metodología utilizada es el meta-análisis de los resultados estadísticos globales proporcionados por la Consejería de Educación, Cultura y Deporte. La muestra $(\mathrm{N}=$ 36.512) la compone el total del alumnado de los niveles indicados que participaron en las pruebas. Los resultados obtenidos apuntan que el enfoque por competencias ha tenido repercusiones positivas en el rendimiento escolar del alumnado y se constata que las variables individuales y el nivel socioeconómico y cultural del alumnado ejercen una influencia diferencial. Este estudio pone de manifiesto que las evaluaciones de diagnóstico aportan indicadores claves a los equipos directivos para orientar el diseño e implementación de unos planes de mejora desde los que se promuevan unas prácticas docentes que contribuyan al desarrollo de las competencias básicas e incidan en el cambio cultural de los centros educativos.

\section{Palabras clave:}

Competencias básicas; características del alumnado; características sociales; resultados escolares. by students taking the fourth year of Primary Education and the second year of Secondary Education in the diagnostic evaluations in Asturias in the last six academic years (2006-2012), in order to know the impact that the competency-based approach has had on educational outcomes of students in this region. The method used was a meta-analysis of the overall statistical results provided by the Department of Education, Culture and Sport. The sample $(\mathrm{N}=36,512)$ comprises all the students of those years who participated in the evaluations which were conducted through the completion of the several instruments designed for each academic year (ad-hoc questionnaires). The results show that the competency-based approach has had positive repercussions on the students' school performance and that the individual variables and the socioeconomic and cultural level of the students have exerted a differential influence. This study proves that diagnostic evaluations provide key indicators for the management of teams so that they can guide the design and implementation of the improvement plans in order to foster teaching practices that can help develop the basic competencies and lead to a cultural change at schools.

\section{Key words:}

Keys competences; student characteristics; social characteristics; outcomes of education.

\section{Résumé:}

Les nouvelles lois régulant le système éducatif en Espagne coïncident en deux aspects: l'incorporation des Compétences Basiques et l'implantation des évaluations de diagnostic pour connaître leur degré de développement. L'application de ces épreuves dans la Principauté des Asturies depuis l'année scolaire 2005-2006 a fait de cette région une zone pionnière pour leur conception et leur développement. Dans cet article nous présentons une méta-analyse des résultats obtenus par les élèves de quatrièmes année d'école primaire (cours moyen 1) et de deuxième année de la E.S.O. (classe de 4 ème [collège]) lors des évaluations de diagnostic pendant les six dernières années académiques (2006-2012), dans le but de connaitre l'impact que l'approche par compétences a eu dans les résultats scolaires de cette région. La méthodologie utilisée est celle de la méta-analyse des résultats statistiques globaux fournis par le Département Régional de l'Éducation, de la Culture et du Sport. L'échantillon $(\mathrm{N}=36.512)$ est composé par la totalité des élèves des niveaux indiqués ayant participé aux épreuves. Les résultats obtenus signalent que l'approche par compétences a eu des répercussions positives dans les performances scolaires des élèves, et il est constaté que les variables individuelles et le niveau socioéconomique et culturel des élèves exercent une influence différentielle. Cet- 
José Antonio Álvarez Castrillón, Alejandro Rodríguez-Martín y Emilio Álvarez-Arregui

te étude met en évidence que les évaluations de diagnostic fournissent des indicateurs clé aux équipes de direction pour orienter la conception et l'implémentation de plans d'amélioration à partir desquels des pratiques d'enseignement contribuant au développement des compétences basiques sont promues et qui causent des changements culturel des centres d'enseignement.

\section{Mots clés:}

Compétences Basiques; caractéristiques des élèves; caractéristiques sociales; résultats scolaires.

Fecha de recepción: 30-1-2014

Fecha de aceptación: 14-5-2014

\section{Introducción}

Desde hace más de dos décadas los sistemas educativos europeos han venido experimentando importantes reajustes como consecuencia de las crecientes demandas socioeconómicas para que se forme a personas capaces de desenvolverse en entornos dinámicos de complejidad creciente. Es por ello que los gobiernos se han tenido que esforzar en la identificación, diseño y conceptualización de las competencias que debe alcanzar cada estudiante al final de la educación obligatoria según los estándares educativos (OCDE, 2010).

La progresiva incorporación de las competencias en los marcos legales ha ido generando cambios de diferente intensidad que se han traducido en la práctica en un cambio de enfoque en el diseño, desarrollo y evaluación de los currículos escolares. En este punto no podemos perder de vista que aunque la historia de las competencias en nuestro contexto cultural se inicia en la década de los setenta fue habitual su empleo en la Formación Profesional surgida en torno a la LOGSE, lo verdaderamente novedoso, como apuntan Tiana (2011) y De la Orden (2011), ha sido adecuar el concepto de "competencia" a la educación general y a la básica en particular. A esta transferencia contribuyó el Informe "La Educación encierra un tesoro" (Delors, 1996) de la UNESCO, un referente clave para promover la necesidad de que las personas se formen a lo largo de toda la vida atendiendo a cuatro pilares fundamentales: aprender a conocer, aprender a hacer, aprender a vivir juntos y aprender a ser.

El Proyecto DeSeCo (Definición y Selección de Competencias) realizado por la OCDE (2005) y el Programa Internacional para la Evaluación de Estudiantes (PISA) en 1997, son dos documentos que contribuyeron 
a la extensión de las competencias a nivel internacional. El primero establecía un marco para la definición, selección y evaluación de competencias y sus resultados sirvieron para fundamentar las pruebas PISA. A partir de aquí, se generalizan unos procedimientos de evaluación de estas competencias que tendrán su reconocimiento en los respectivos marcos legales de los Estados de la Unión Europea.

\section{El enfoque por competencias: Diversidad terminológica y concreción legal}

Diversos autores han constatado que el término "competencia" ha sido objeto de variados y hasta contradictorios intentos de definición (Perrenound, 1997; Monereo, 2003; Sarramona, 2004; Pérez Gómez, 2007), sin embargo, en todos ellos se identifica un denominador común dual que permite abordar esta diversidad terminológica (Lévy Leboyer, 2003). Así, las competencias se entienden, por un lado, como un conjunto de acciones/decisiones que una persona puede adoptar y, por el otro, hacen referencia al modo en que una persona utiliza su capacidad de decisión de manera satisfactoria. Estamos, por tanto, ante una concepción constructivista de las competencias donde el aprendizaje es abordado desde una perspectiva (Álvarez, Pérez y Suárez, 2008) social, interactiva y, propiamente, constructiva llegándose a denominar a esta triada "Pedagogía de la integración" (Roegiers, 2004) desde el momento que articula aprendizajes donde concurren aprendizajes ya apuntados por el Informe Delors.

La divergencia y la controversia acerca de las competencias se han incrementado con las aportaciones de las instituciones. Así, la OCDE entiende que las competencias son un concepto amplio que puede componerse de habilidades, actitudes y conocimientos (Ananiadou y Claro, 2009) que una persona puede movilizar para hacer frente a situaciones complejas y de la vida diaria. Por tanto, se asume que una competencia no está limitada a elementos cognitivos (uso de la teoría, conceptos o conocimiento implícito) pues abarca aspectos funcionales (habilidades técnicas), atributos interpersonales (habilidades sociales u organizativas) y valores éticos (Rychen y Hersch, 2003).

La UE también ha trascendido el plano conceptual en sus análisis y propuestas y, por ello, el Consejo Europeo de Lisboa (2000) conclu- 
José Antonio Álvarez Castrillón, Alejandro Rodríguez-Martín y Emilio Álvarez-Arregui

yó que un marco de referencia europeo debía definir las nuevas cualificaciones básicas que debe proporcionar el aprendizaje permanente, situándolo como medida ante la globalización y el desplazamiento hacia las economías basadas en el conocimiento. Desde entonces, las conclusiones de este Consejo se fueron refrendando en las diferentes sesiones del órgano, cristalizando en 2004 con el Informe conjunto que el Consejo y la Comisión adoptaron sobre el programa de trabajo "Educación y Formación 2010" que analizaba en profundidad la necesidad de garantizar sistemas educativos que promuevan la adquisición de las competencias clave para el aprendizaje permanente. Además, en este programa, se publicó como "Recomendación del Parlamento Europeo y del Consejo de 18 de diciembre de 2006 sobre las competencias clave para el aprendizaje permanente" dónde se recoge por primera vez la concreción semántica y operativa de las ocho competencias clave que configuran el marco de referencia europeo:

- comunicación en la lengua materna,

- comunicación en lenguas extranjeras,

- competencia matemática y competencias básicas en ciencia y tecnología,

- competencia digital,

- aprender a aprender,

- competencias sociales y cívicas,

- sentido de la iniciativa y espíritu de empresa, y

- conciencia y expresión culturales

En las instancias comunitarias siempre se ha tratado de distinguir "habilidad" - capacidad de realizar tareas y solucionar problemas- y "competencia" - capacidad de aplicar los resultados del aprendizaje en un determinado contexto educativo, laboral, personal, etc. (Cedefop, 2008). Sin embargo, dado que los términos se usan indistintamente y las definiciones difieren según los distintos países y regiones, la delimitación conceptual y asociativa con otros conceptos relacionados no ha quedado clara (Climént, 2010; Ribes, 2011). Autores como Oliveros Martín-Vares (2006) y Jurado Valencia (2009) consideran que las competencias no se enseñan o aprenden, sino que se desarrollan, debiendo distinguirse entre el desarrollo de competencias asociadas al puesto de trabajo y la formación en competencias asociadas a la actividad de los centros educativos, es decir, debe diferenciarse entre competencias académicas y 
profesionales en los procesos de concreción curricular y de evaluación de la educación.

La diversidad terminológica respecto a las competencias también nos obliga a remitirnos al marco normativo de manera singular. En España se sitúa originalmente en la Ley Orgánica 10/2002, de 23 de diciembre, de Calidad de la Educación (LOCE) si bien su mayor impacto se ha producido a partir de la Ley Orgánica 2/2006, de 3 de mayo, de Educación (LOE) al quedar incorporadas las competencias básicas como uno de los elementos del currículo coexistiendo con objetivos, contenidos y criterios de evaluación. Además, con la LOE las competencias se constituyen en referente para la promoción en la Educación Primaria, la obtención del Graduado en Educación Secundaria Obligatoria y la realización de las evaluaciones de diagnóstico.

De acuerdo con el marco europeo, en la concreción del currículo tras la implantación de la LOE, se identifican las ocho competencias básicas de nuestro sistema educativo, cuya descripción, finalidad y aspectos quedan recogidos en los Reales Decretos 1513/2006 y 1631/2006 de Educación Primaria y E.S.O. respectivamente donde se relaciona cada competencia con las áreas y materias de las etapas educativas que integran la educación básica. Esta propuesta curricular, en el caso del Principado de Asturias, se concreta en el Decreto 56/2007 (BOPA n 140 de 16.06.2007) y el Decreto 74/2007 (BOPA $n^{\circ} 162$ de 12.07.2007) por los que se ordenan y establecen, respectivamente, los currículos de la Educación Primaria y Secundaria.

En los diferentes niveles de concreción curricular se comprueba que las competencias tienen un carácter central en la programación de la enseñanza, en la selección de actividades de aprendizaje y en la evaluación del alumnado, algo lógico dado que debe garantizarse que al término de la educación básica hayan adquirido aquellos aprendizajes que se consideran imprescindibles para su realización personal, inclusión social y ciudadanía activa, así como para prepararlos para la vida adulta y el aprendizaje permanente. De ahí que la evaluación de las competencias básicas, a pesar de las dificultades que implica (Castro, 2010), adquiera un papel relevante al trasladar el foco de atención desde los contenidos aprendidos a la capacidad para utilizar los aprendizajes en diversas situaciones vitales (Viso Alonso, 2010). 


\section{Las evaluaciones de diagnóstico como referente pedagógico para el cambio}

La legislación educativa reciente en España viene haciendo referencia a este tipo de evaluaciones consecuencia, en buena medida, de la influencia de los programas de evaluación educativa más reputados con marcos teóricos consistentes y evidencias de fiabilidad y validez caso del Programme for International Student Assessment (PISA), Trends in International Mathematics and Science Study (TIMSS), Progress in International Reading Literacy Study (PIRLS) o National Assessment of Educational Progress (NAEP).

En la LOCE (Arts. 18 y 30) se estableció una evaluación general de diagnóstico para comprobar el grado de adquisición de las competencias básicas en Educación Primaria y en Educación Secundaria Obligatoria. En ambos casos la evaluación no tenía efectos académicos sino informativos y orientadores para los centros, el profesorado, las familias y el alumnado.

La LOE (Arts. 21 y 29) recoge diversos modelos de evaluación del sistema educativo entre los que se incorpora las evaluaciones de diagnóstico del alumnado al finalizar el segundo ciclo de Educación Primaria $\left(4^{\circ}\right.$ curso) y el segundo curso de la E.S.O. Además (Art. 144) se indica que "corresponde a las Administraciones educativas desarrollar y controlar las evaluaciones de diagnóstico en las que participen los centros de ellas dependientes y proporcionar los modelos y apoyos pertinentes a fin de que todos los centros puedan realizar de modo adecuado estas evaluaciones, que tendrán carácter formativo e interno".

Estas pruebas están vinculadas a un enfoque por competencias en cuanto a la implementación del currículo escolar lo que ha supuesto un mayor nivel de exigencia para el profesorado y para el conjunto del sistema educativo. En cualquier caso, al situar las evaluaciones diagnósticas como referente pedagógico se ha buscado una doble finalidad (Collera, 2007), por un lado, orientar mejoras mediante la aportación de datos relevantes para los centros, las familias y la propia administración educativa y, por otro, promover un ejercicio de rendición de cuentas al conjunto de la comunidad educativa, destacando la dimensión informativa que poseen frente al carácter sumativo, acreditativo o final de otras evaluaciones. El objeto final de las evaluaciones de diagnóstico será, por tanto, proporcionar información de interés que facilite el diseño, desa- 
rrollo y evaluación de planes de mejora contextualizados a la realidad de cada centro.

La revisión de la literatura sobre este campo nos muestra que la mayoría de estudios e investigaciones se han centrado, principalmente, en la incorporación de las competencias básicas en las programaciones didácticas, el diseño de tareas y las necesarias adaptaciones que ello implica en las prácticas docentes (Barrero Fernández y Domingo Segovia, 2010; Sierra-Arizmendarrieta, Méndez-Giménez y Mañana-Rodríguez, 2012). Otro aspecto importante objeto de investigación se ha asociado a las dificultades del proceso ya que no se trata de medir resultados exclusivamente sino de diseñar una modalidad de evaluación que, combinando aspectos cuantitativos y cualitativos, permita medir aspectos de carácter reflexivo, actitudinal, situacional y no sólo técnicos (Monereo y Pozo, 2007; Rosales López, 2010; Zabala y Arnau, 2007).

También hay referencias de interés sobre los instrumentos empleados en la evaluación de competencias básicas siempre que se realice un cuidadoso análisis de las fortalezas y debilidades encontradas (Fernández-Alonso y Muñiz, 2011). Las investigaciones sobre la eficacia escolar (Sammons, Thomas y Mortimore, 1997; Murillo, 2007) también destacan la importancia de las evaluaciones de diagnóstico por sus repercusiones en el funcionamiento de los centros educativos y en el rendimiento académico. Desde este marco las evaluaciones diagnósticas constituyen un proceso de recogida y análisis de información amplio sobre los aspectos de los centros educativos que parecen influir de manera directa en el desarrollo de las capacidades del alumnado con el objetivo de promover y avanzar hacia un modelo de calidad y equidad combinado y corresponsable que permita la mejora del sistema educativo en su conjunto. Por ello, se trata de un proceso riguroso, sistemático, con un fin orientativo y formativo que permita comprender mejor la realidad de un centro y de un aula para transformarla.

\section{Método}

Esta investigación trata de conocer el impacto que el enfoque por competencias ha tenido en los resultados escolares del alumnado del Principado de Asturias a través de los datos recogidos en las evaluaciones de diagnóstico realizadas desde 2006 en $4^{\circ}$ curso de Educación Primaria 
y $2^{\circ}$ curso de Educación Secundaria Obligatoria. Con este enfoque se pretende demostrar que a través de las evaluaciones de diagnóstico se puede describir el grado de adquisición de las competencias básicas que tiene el alumnado así como la evolución de este índice durante los seis cursos académicos en los que se vienen realizando estas pruebas tras la implantación de la LOE.

\section{Objetivos}

En este sentido, la finalidad del estudio es aportar información relevante para la mejora del propio sistema educativo en Asturias en cuanto al rendimiento del alumnado y a los factores y variables socioeducativas asociadas a las diferencias en el logro de las competencias básicas. Se pueden resumir los objetivos como sigue:

- Mostrar la evolución en el grado de adquisición de las competencias básicas del alumnado de Asturias.

- Presentar las tendencias más relevantes de los resultados académicos del alumnado de acuerdo a factores individuales.

- Destacar la influencia que las características personales del alumnado y el contexto socioeconómico generan en los resultados académicos del alumnado en la cohorte objeto de estudio.

- Proporcionar a la comunidad educativa y a la administración un análisis de la situación para tomar decisiones orientadas a la mejora del sistema educativo en Asturias.

Atendiendo a los objetivos de la investigación se realiza un metaanálisis que permite hacer síntesis cuantitativas y cualitativas de lo ya investigado, resituar el estado de la cuestión y llegar, o intentar llegar, a una generalización de los resultados obtenidos. El meta-análisis es, por tanto, "una investigación sobre investigaciones" (Morales, 1993, p. 191) que constituye una pieza clave al definir procedimientos de análisis de datos extraídos de los trabajos de investigación revisados, con el fin de obtener conclusiones estadísticas a partir de ellos.

\section{Muestra}

Las características y objetivos de la investigación determinan que la muestra esté compuesta por todo el alumnado participante en las evaluaciones 
de diagnóstico que ha venido realizando la Consejería con competencias educativas en el Principado de Asturias desde el año 2006 hasta el año 2011 y que asciende a un total de 36.512 alumnos/as (Gráfico 1) de los cuales, $16.977(46,50 \%)$ corresponden al $4^{\circ}$ curso de Educación Primaria y $19.535(53,50 \%)$ al $2^{\circ}$ curso de E.S.O. Cada curso escolar las pruebas se han realizado en un solo curso o, también, en ambos cursos simultáneamente y atendiendo a diferentes áreas y competencias evaluadas.
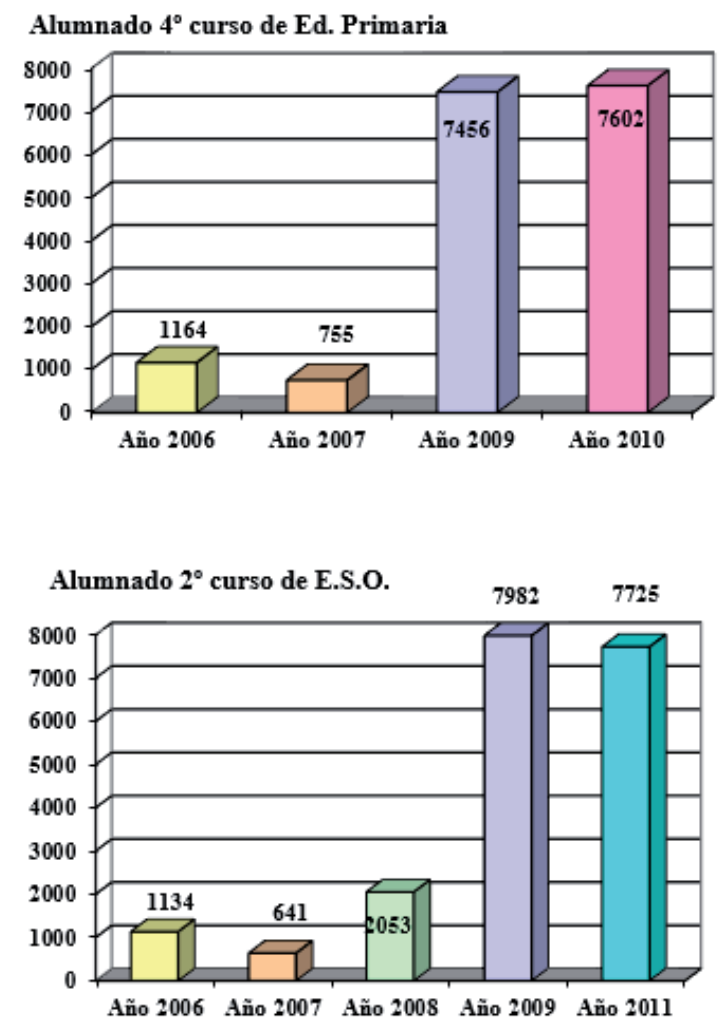

\section{Gráfico 1. Alumnado participante en las evaluaciones de diagnóstico}

Las evaluaciones de diagnóstico en Asturias se iniciaron de manera experimental en el año 2005 y a partir de ahí se han realizado diversas aplicaciones. En el año 2009 se desarrollará una evaluación amplia sobre las poblaciones de $4^{\circ}$ de Educación Primaria y $2^{\circ}$ de E.S.O. simultáneamente el mismo curso académico y valorando competencias diferentes.

En la Tabla 1 se recogen los datos del meta-análisis, en concreto, el año, el curso que participó en cada evaluación diagnóstico, los centros 
y el alumnado participante, así como las áreas y competencias objeto de estudio. En la penúltima columna (d) presentamos el resultado global de cada estudio respecto al valor añadido o eficacia relativa ${ }^{3}$, es decir, la capacidad de los centros educativos para mejorar los resultados que, en función de su entorno sociocultural, se puede esperar de su alumnado, expresada como diferencia media tipificada.

Los valores positivos de este indicador señalan que el alumnado logra mejores resultados de lo esperado por su nivel socioeconómico y cultural.

Tabla 1. Características y resultados de cada estudio de meta-análisis

\begin{tabular}{|c|c|c|c|c|}
\hline $\begin{array}{l}\text { Estudio } \\
\text { Año }\end{array}$ & Curso & Centros Alum & & $\begin{array}{l}\text { Áreas o Competencias objeto de } \\
\text { evaluación }\end{array}$ \\
\hline \multirow{2}{*}{2006} & $4^{\circ}$ E.P. & 1164 & 0,63 & \multirow{2}{*}{$\begin{array}{l}\text { Comunicación lingüística en cas- } \\
\text { tellano e inglés } \\
\text { Matemáticas }\end{array}$} \\
\hline & $2^{\circ}$ E.S.O. 2 & 1134 & 0,78 & \\
\hline \multirow{2}{*}{2007} & $4^{\circ}$ E.P. $\quad 1$ & 755 & 0,65 & \multirow{2}{*}{$\begin{array}{l}\text { Comunicación lingüística en cas- } \\
\text { tellano e inglés } \\
\text { Matemáticas }\end{array}$} \\
\hline & $2^{\circ}$ E.S.O. 9 & 641 & 0,61 & \\
\hline
\end{tabular}

Comunicación lingüística en castellano e inglés

Matemática $2008 \quad 2^{\circ}$ E.S.O.30 $2053 \quad$ 0,62 $\begin{aligned} & \text { Conocimiento e interacción con el } \\ & \text { mundo físico }\end{aligned}$

Competencia social y ciudadana

Competencia en aprender a aprender

Comunicación lingüística en inglés

$4^{\circ}$ E.P. $\quad 3097456 \quad 0,65$ Competencia social y ciudadana

Competencia en aprender a aprender

2009 Comunicación lingüística en castellano e inglés

$2^{\circ}$ E.S.O.148 $7982 \quad 0,60$ Matemática

Conocimiento e interacción con el mundo físico

3 Se trata de un indicador de calidad en la medida en que da a entender en qué grado los resultados logrados por el alumnado se corresponden con lo que cabría esperar de las particulares condiciones del entorno de los centros, En otras palabras, se puede entender como la capacidad de los centros escolares para mejorar los resultados que, en función de su entorno sociocultural, se pueden esperar de su alumnado. 
Enfoque por competencias y resultados escolares. Estudio longitudinal en el Principado de Asturias

José Antonio Álvarez Castrillón, Alejandro Rodríguez-Martín y Emilio Álvarez-Arregui

\begin{tabular}{lrrr}
\hline $2010 \quad 4^{\circ}$ E.P. $298 \quad 7602$ & $0,64 \begin{array}{l}\text { Matemática } \\
\text { Conocimiento e interacción con el } \\
\text { mundo físico }\end{array}$ \\
\hline $2011 \quad 2^{\circ}$ E.S.O.148 & $7725 \quad 0,62 \begin{array}{l}\text { Competencia Matemática } \\
\text { Conocimiento e interacción con el } \\
\text { mundo físico }\end{array}$ \\
\hline
\end{tabular}

Fuente: Adaptación de Consejería de Educación y Ciencia del Principado de Asturias (2010)

\section{Instrumentos y Procedimiento}

Los instrumentos utilizados para el meta-análisis han sido los informes publicados durante los últimos seis años por la Consejería de Educación donde se recogen los datos resultantes de las evaluaciones de diagnósticos realizadas cada curso académico.

El modelo teórico de las evaluaciones de diagnóstico en Asturias contempla factores de contexto o entrada al sistema educativo sobre los que los centros tienen escasa o nula posibilidad de actuación: los factores de proceso escolar sobre las que el centro puede intervenir directamente y los resultados educativos individuales que no son independientes de los factores anteriores. Por ello, una de las finalidades básicas de la evaluación de diagnóstico es identificar los factores ambientales que potencialmente se asocian a buenos resultados académicos y analizar la relación entre dichos factores y la puntuación alcanzada en las pruebas de evaluación. De esta forma para realizar el análisis se aplican diferentes cuestiones que son cumplimentados por el profesorado, los equipos directivos, las familias y el alumnado participante que, en esta investigación, constituyen la muestra de estudio.

Al igual que en la mayoría de evaluaciones nacionales e internacionales, la Evaluación de Diagnóstico de Asturias utiliza, para el análisis de los resultados, el método conocido como Teoría de Respuesta al Ítem (TRI), que permite situar el nivel de competencia de cada alumno/a en una escala común donde se ubica al alumnado a lo largo de un continuo de competencia con seis niveles de rendimiento (Tabla 2$)^{4}$ en cada una de las competencias evaluadas describiendo conocimientos, habilidades y actitudes que muestran las alumnas y los alumnos al responder a las pruebas. A cada nivel se le atribuye un intervalo de puntos.

4 Los niveles de rendimiento describen los conocimientos, habilidades y actitudes. Son progresivos y se expresan en un rango de puntuación de menor a mayor que se corresponde con un grado de adquisición de la competencia evaluada. 
José Antonio Álvarez Castrillón, Alejandro Rodríguez-Martín y Emilio Álvarez-Arregui

Tabla 2. Niveles de rendimiento en la Evaluación de Diagnóstico

\begin{tabular}{|c|c|c|c|c|c|}
\hline Nivel 1 & Nivel 2 & Nivel 3 & Nivel 4 & Nivel 5 & Nivel 6 \\
\hline Inferior & Bajo & Medio-Bajo & Medio-Alto & Alto & Superior \\
\hline $\begin{array}{l}\text { por debajo } \\
\text { del percen- } \\
\text { til } 10\end{array}$ & $\begin{array}{l}\text { entre los } \\
\text { percentiles } \\
10 \text { y } 25\end{array}$ & $\begin{array}{l}\text { entre los } \\
\text { percentiles } \\
25 \text { y } 50\end{array}$ & $\begin{array}{l}\text { entre los } \\
\text { percentiles } \\
50 \text { y } 75\end{array}$ & $\begin{array}{l}\text { entre los } \\
\text { percentiles } \\
75 \text { y } 90\end{array}$ & $\begin{array}{l}\text { por encima } \\
\text { del percen- } \\
\text { til } 90\end{array}$ \\
\hline $\begin{array}{l}\text { Menos de } \\
357 \text { puntos }\end{array}$ & $\begin{array}{l}\text { Entre } 358- \\
447 \text { puntos }\end{array}$ & $\begin{array}{l}\text { Entre } 448- \\
501 \text { puntos }\end{array}$ & $\begin{array}{l}\text { Entre } 502- \\
572 \text { puntos }\end{array}$ & $\begin{array}{l}\text { Entre } 573- \\
624 \text { puntos }\end{array}$ & $\begin{array}{l}\text { Más de } 624 \\
\text { puntos }\end{array}$ \\
\hline
\end{tabular}

Fuente: Adaptación de Consejería de Educación y Ciencia del Principado de Asturias (2010)

Tras obtener la información de las evaluaciones de diagnóstico realizadas desde la Dirección General de Formación Profesional, Desarrollo Curricular e Innovación Educativa de la Consejería de Educación, Cultura y Deporte del Principado de Asturias, el procedimiento a seguir para evitar los sesgos de publicación y autor ha sido el empleo de las estrategias generales aplicadas a la metodología meta-analítica (Marín, Sánchez, Huedo y Fernández, 2007): 1. Promediar resultados a través de los estudios, 2. Evaluar la heterogeneidad de los resultados y 3 . Formular modelos explicativos de la heterogeneidad en caso de confirmarse, en función de las características diferencias de los estudios analizados. Además, el parámetro de medida que empleado para combinar los diferentes estudios es la diferencia de proporciones entre los grupos analizados.

\section{Resultados}

Los resultados se organizan en tres apartados que responden a los tres primeros objetivos formulados en esta investigación. En primer lugar se presenta la evolución en el grado de adquisición de las competencias básicas evaluadas en cada curso académico. Posteriormente, se presentan los resultados de los factores individuales que influyen en los resultados escolares (el sexo, el autoconcepto académico, las expectativas académicas y la permanencia de un año más en un curso académico.). Finalmente se analiza cómo se relacionan las variables de carácter contextual (índice socioeconómico cultural, apoyo familiar y la valoración del contexto del centro educativo). 


\section{Grado de adquisición de las competencias básicas}

A continuación se recogen (Gráficos 2 y 3) los principales datos de la evolución en cuanto al grado de adquisición de determinadas competencias objeto de análisis a través de las evaluaciones de diagnóstico.

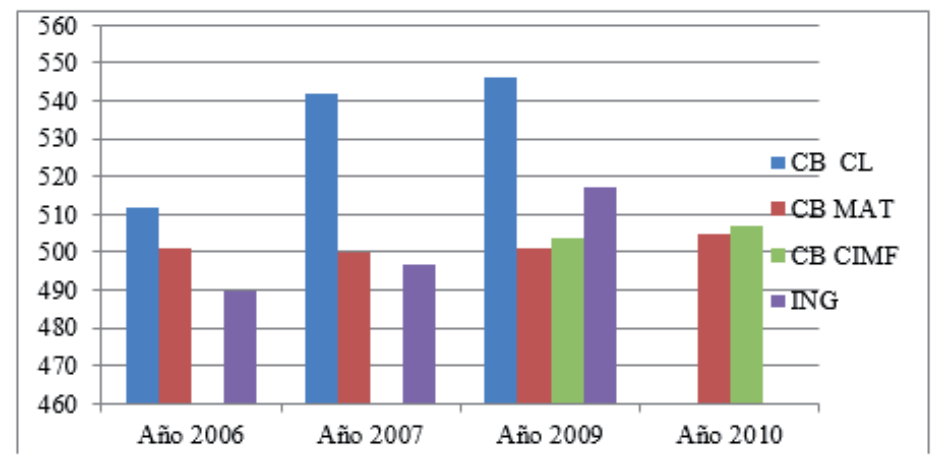

Gráfico 2. Distribución de resultados por el logro de competencias en $4^{\circ} \mathrm{de}$ Ed. Primaria

Fuente: Elaboración propia

Leyenda: Competencia en comunicación lingüística en Castellano $(\mathrm{CB} \mathrm{CL})$;

Competencia matemática (CB MAT); Competencia en el conocimiento y la interacción con el mundo físico (CB CIMF); Comunicación lingüística en Inglés ING

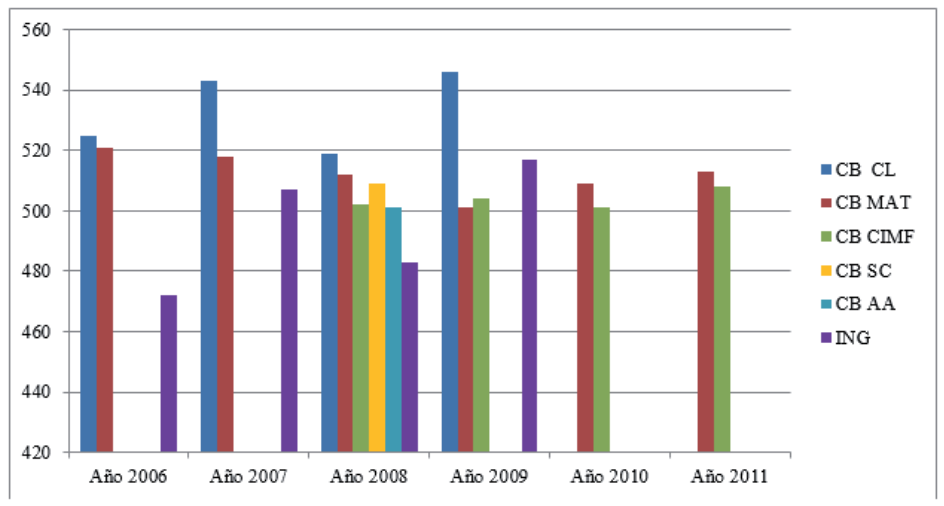

Gráfico 3. Distribución de resultados por el logro de competencias en $2^{\circ}$ de E.S.O. Fuente: Elaboración propia

Leyenda: Competencia en comunicación lingüística en Castellano $(\mathrm{CB} \mathrm{CL})$; Competencia matemática (CB MAT); Competencia en el conocimiento y la interacción con el mundo físico (CB CIMF); Competencia social y ciudadana (CB SC); Competencia para aprender a aprender (CB AA); Comunicación lingüística en Inglés (ING) 
José Antonio Álvarez Castrillón, Alejandro Rodríguez-Martín y Emilio Álvarez-Arregui

Aunque las áreas y competencias evaluadas durante los seis cursos académicos no han sido las mismas, se puede observar una tendencia que se mantiene positiva en las competencias que sí han sido evaluadas de manera continuada. Este es el caso, por ejemplo, tanto en Educación Primaria como en ESO, la Competencia Matemática ha ido obtenido mejores resultados paulatinamente.

\section{Factores individuales}

En cuanto a los factores individuales, el modelo de evaluación diagnóstico de Asturias plantea una serie de variables que permiten analizar el impacto que tiene en el desarrollo de las competencias y en el rendimiento académico el sexo (Gráfico 4), el autoconcepto académico (Gráfico 5), la permanencia de un año más en un curso académico (Gráfico 6) y, por último, las expectativas académicas sólo en el alumnado que cursa $2^{\circ} \mathrm{de}$ ESO (Gráfico 7). Las tendencias en este sentido se han revelado similares, sin que se describan diferencias, tanto en Educación Primaria como en ESO. Por ello, la presentación de los resultados se realiza atendiendo a la distribución de las medidas de las variables indicadas pero sólo en cuatro competencias que durante estos seis años han tenido continuidad y, al menos, han sido evaluadas en tres cursos (Comunicación lingüística en castellano (CB CL), Competencia Matemática (CB MAT), Competencia en el conocimiento y la interacción con el mundo físico (CB CIMF) y Comunicación lingüística en inglés (CB ING). En cada una de estas competencias se muestran las puntuaciones medidas obtenidas en cada curso académico, si bien, en los cursos donde no se reflejan resultados corresponden a evaluaciones diagnósticas donde dicha competencia no fue objeto de estudio con carácter general o bien la evaluación sólo correspondió a una etapa educativa.

El sexo constituye, como puede observarse en los gráficos una variable que permite identificar diferencias. En este caso las alumnas de $4^{\circ}$ de E.P. y E.S.O. obtienen mejores puntuaciones en las competencias analizadas, destacando como resultados más óptimos los obtenidos en la Competencia Lingüística. Cabe destacar, también, que en la Competencia Matemática tradicionalmente las alumnas obtienen resultados inferiores a los alumnos, situación que vuelve a confirmarse, en este estudio de manera general. 
Enfoque por competencias y resultados escolares. Estudio longitudinal en el Principado de Asturias

José Antonio Álvarez Castrillón, Alejandro Rodríguez-Martín y Emilio Álvarez-Arregui

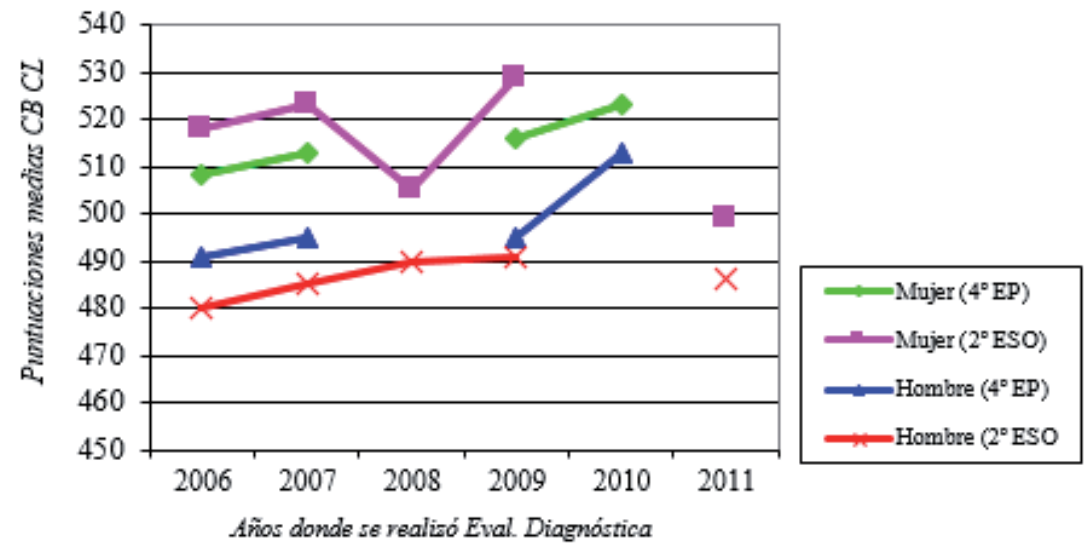

Fuente: Elaboración propia

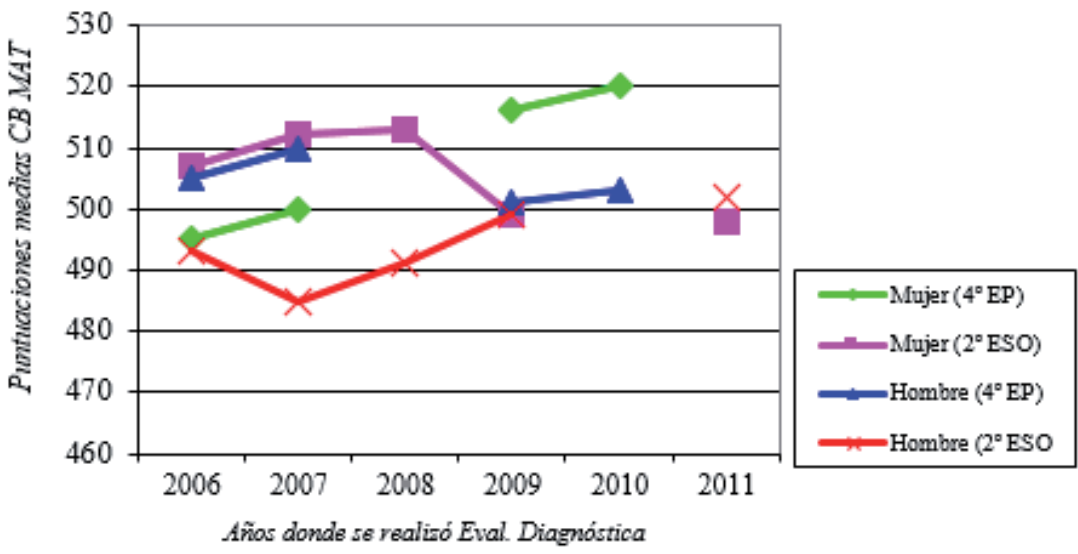

Fuente: Elaboración propia

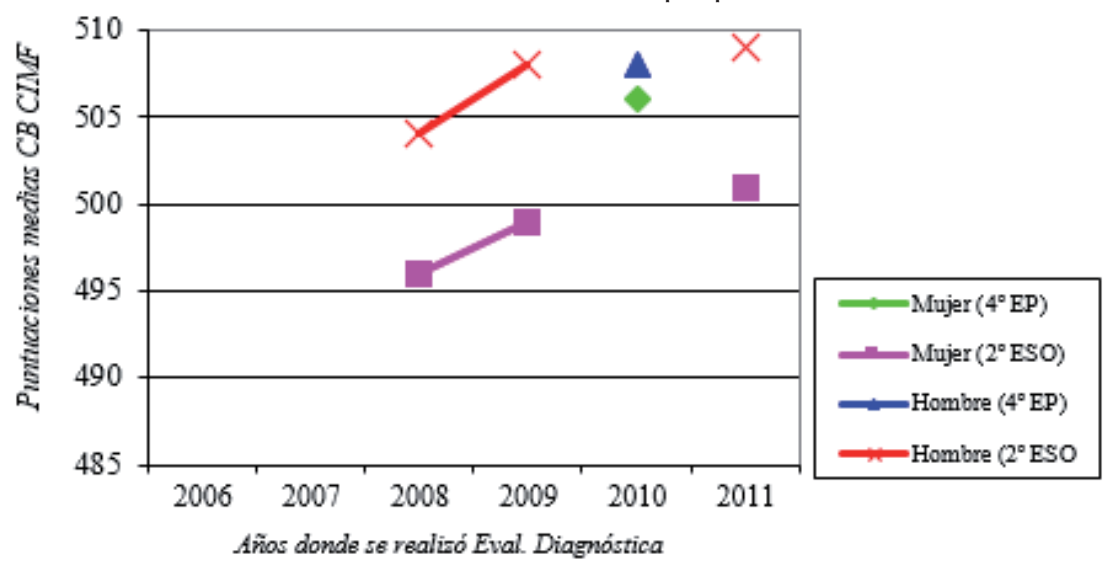

Fuente: Elaboración propia 


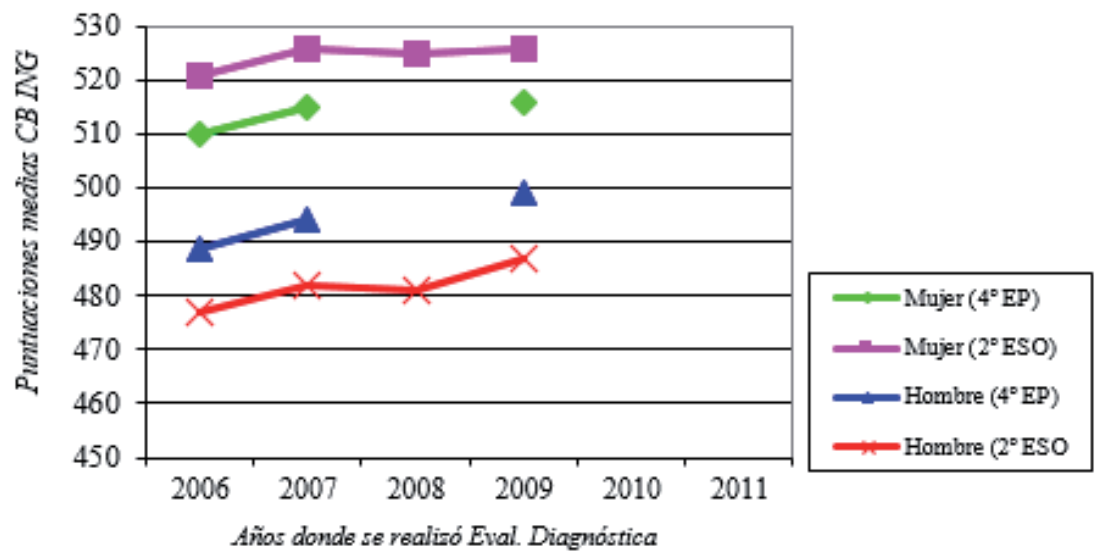

Fuente: Elaboración propia

\section{Gráfico 4. Distribución de medias en relación con el sexo y resultados por competencias}

El autoconcepto académico ${ }^{5}$, es decir, cómo se valora a si mismo cada alumno/a, es el índice resultante de un conjunto de preguntas de la evaluación diagnóstica que permite caracterizar al alumnado participante. Este índice permite dibujar un perfil de autoconcepto (bajo, parcial, bueno y alto) y comprobar que constituye una variable que correlaciona con los resultados globales obtenidos en las competencias analizadas. De esta forma, el rendimiento se incrementa a medida que el autoconcepto es mayor, y es en la Competencia Matemática y en la Competencia Lingüística en Inglés donde se muestra de forma más clara y sostenida en los años analizados y cómo las tendencias invertidas "autoconcepto alto-autoconcepto bajo" aumentan su distancia a lo largo de los años.

5 A partir de algunos ítems de la encuesta del alumnado se construyó un índice que resume el autoconcepto académico del alumnado. Los ítems estaban redactados como afirmaciones y el alumnado debía valorar el grado de anuencia con las mismas en una escala de 0 a 3 puntos, donde el 0 significaba nunca o casi nunca y el 3 siempre o casi siempre. 
Enfoque por competencias y resultados escolares. Estudio longitudinal en el Principado de Asturias

José Antonio Álvarez Castrillón, Alejandro Rodríguez-Martín y Emilio Álvarez-Arregui
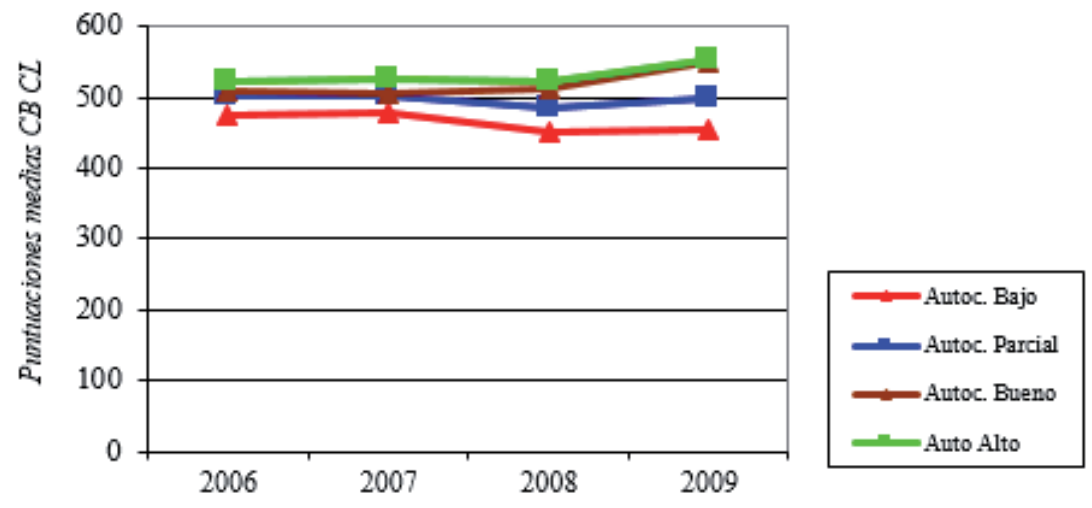

Años donde se realizó Eval. Diagnóstica

Fuente: Elaboración propia

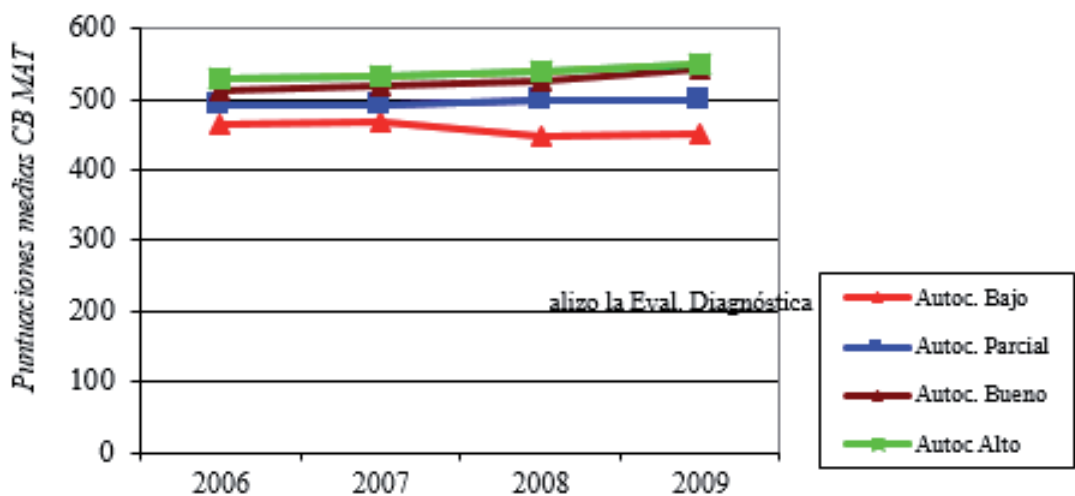

Añas donde se realizó Eval. Diagnóstica

Fuente: Elaboración propia

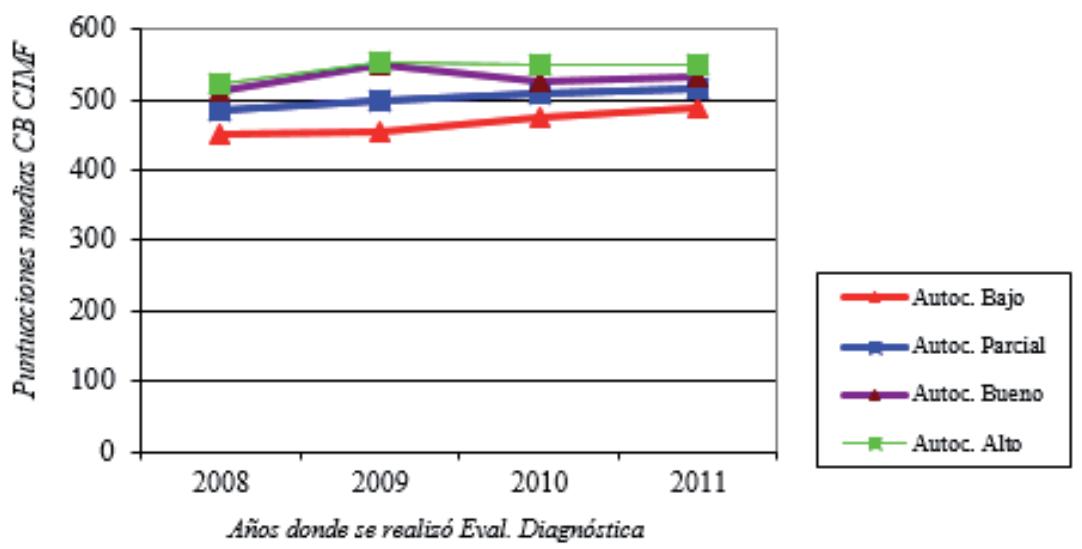

Fuente: Elaboración propia 


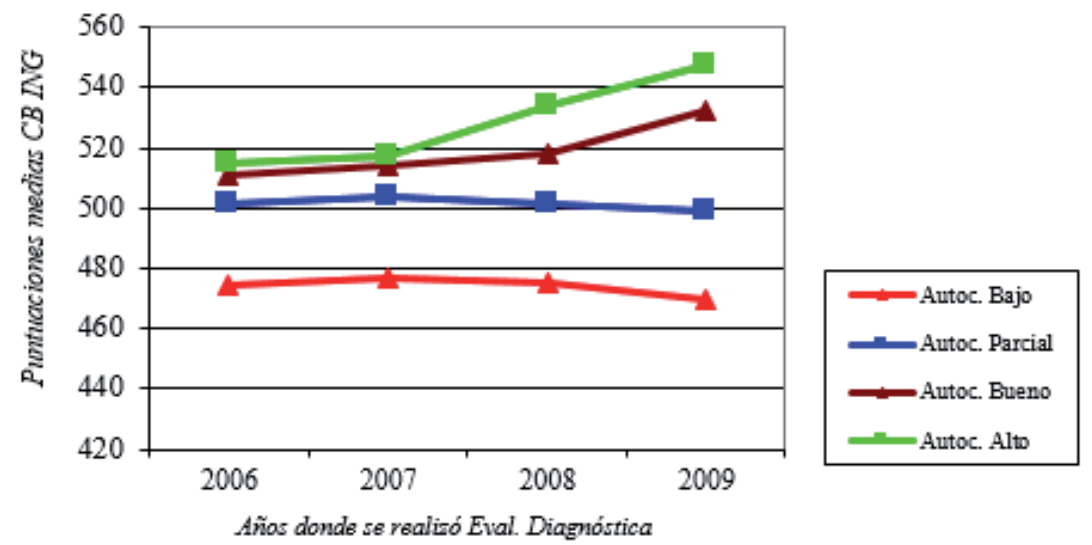

Fuente: Elaboración propia

\section{Gráfico 5. Distribución de medias en relación con el autoconcepto académi- co y resultados por competencias.}

Otra de las variables individuales que influyen en los resultados escolares y, por tanto, en el rendimiento global de las competencias son las expectativas que tiene el alumnado de $2^{\circ}$ de ESO al finalizar la etapa: finalizar la etapa, cursar FP, cursar Bachillerato o acceder a estudios superiores. Se observa de manera constante en toda la cohorte que cuanto mayor es la expectativa académica sobre el futuro, mayor es el promedio de resultados en cuanto al rendimiento en las competencias.

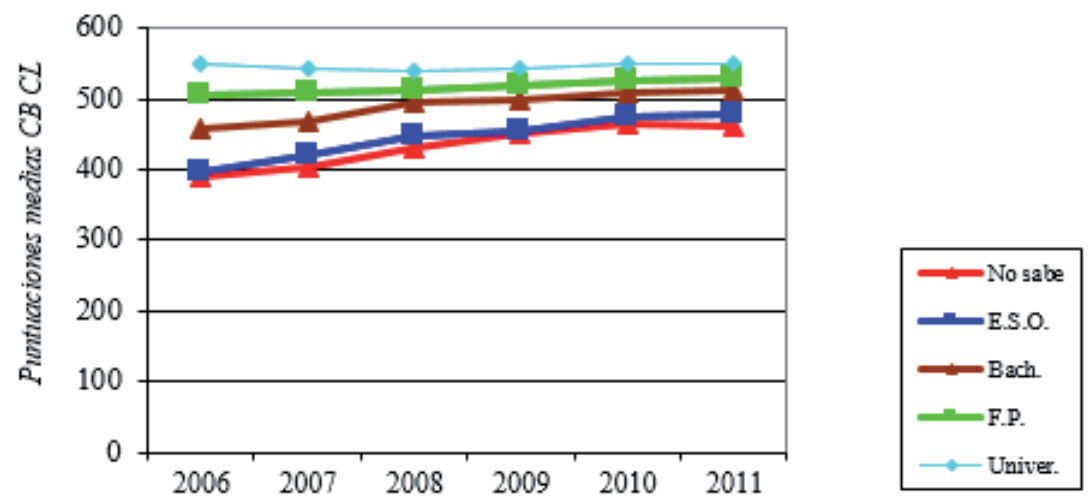

Fuente: Elaboración propia 
Enfoque por competencias y resultados escolares. Estudio longitudinal en el Principado de Asturias

José Antonio Álvarez Castrillón, Alejandro Rodríguez-Martín y Emilio Álvarez-Arregui
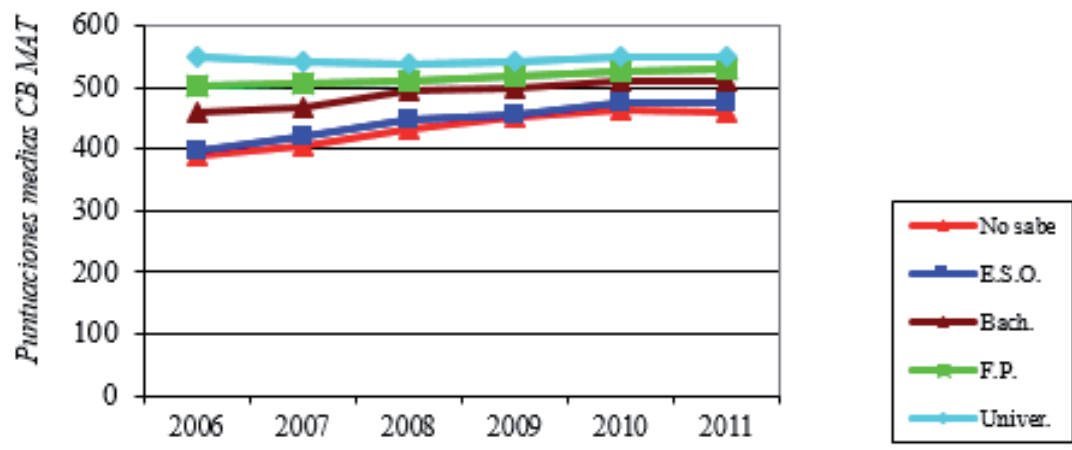

Años donde se realizó Eval. Diagnóstica

Fuente: Elaboración propia

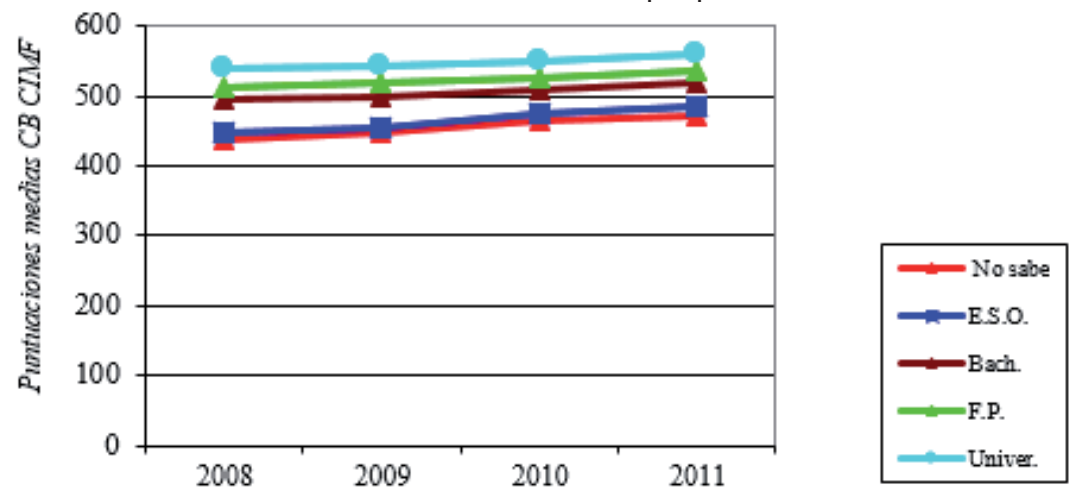

Años donde se realizó Eval. Diagnóstica

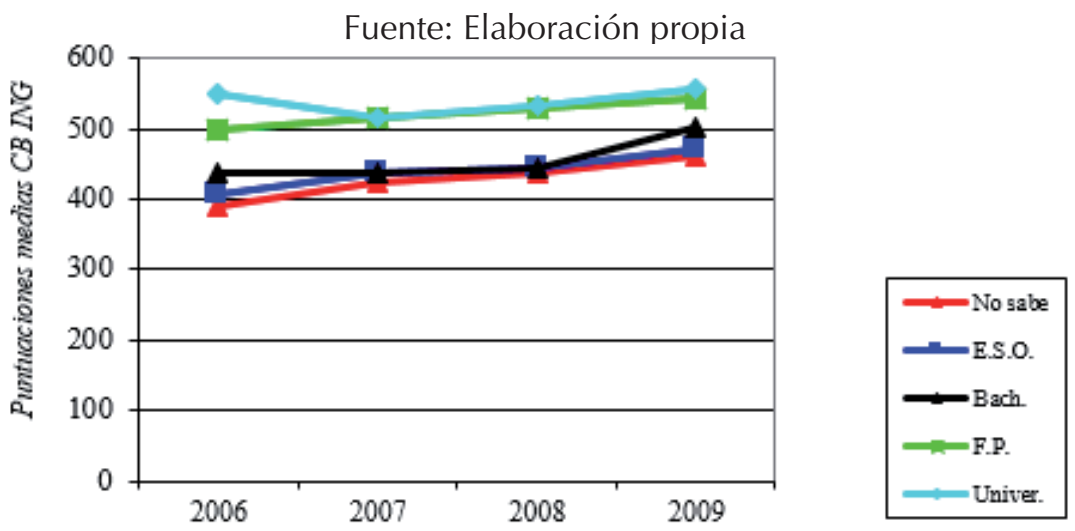

Años donde se realizó Eval. Diagnóstica

Fuente: Elaboración propia

Gráfico 6. Distribución de medias en relación con las expectativas académicas y resultados por competencias 
La permanencia de un año más de un/a alumno/a en un curso no correspondiente a su edad evidencia en todos los casos analizados puntuaciones más bajas en cuanto al logro de las competencias. Destacar, por ejemplo, que el rendimiento en la Competencia Lingüística en castellano y en inglés, desciende progresivamente en el alumnado que permanece en el curso.
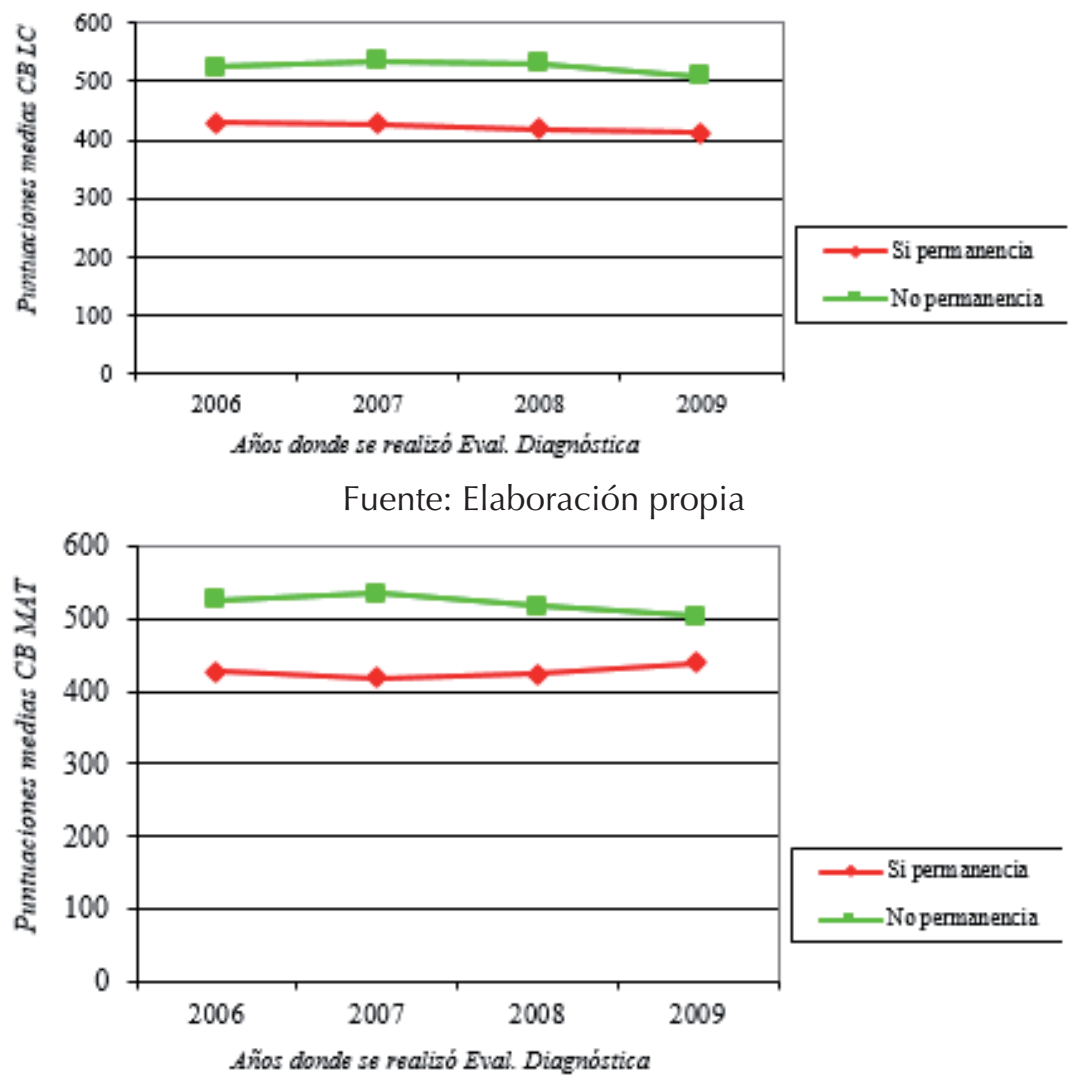

Fuente: Elaboración propia 
Enfoque por competencias y resultados escolares. Estudio longitudinal en el Principado de Asturias

José Antonio Álvarez Castrillón, Alejandro Rodríguez-Martín y Emilio Álvarez-Arregui

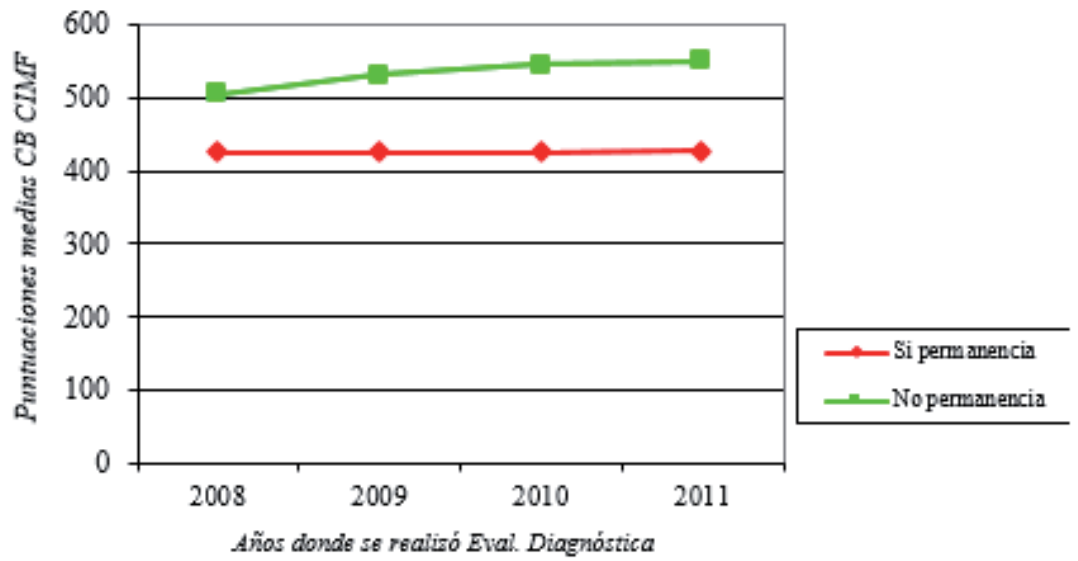

Fuente: Elaboración propia

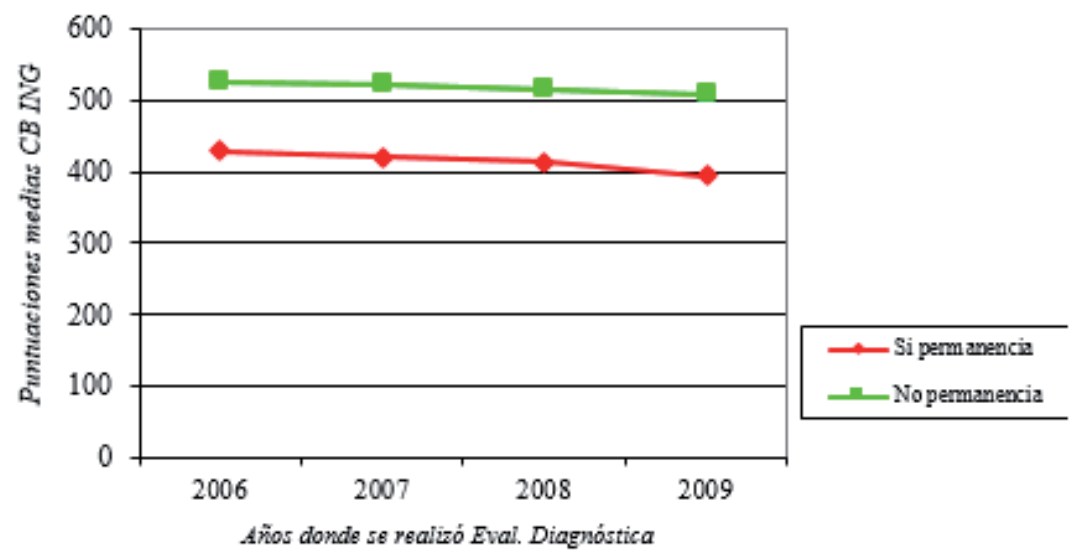

Fuente: Elaboración propia

Gráfico 7. Distribución de medias en relación con la permanencia un año más en el curso académico y resultados por competencias

\section{Variables de carácter contextual}

Las variables contextuales que se han analizado respecto al contexto, en línea con el modelo de evaluación diagnóstico de Asturias, son el Índice Socio-económico y Cultural (Gráfico 8) que supone un importante indicador para analizar el grado de influencia que puede tener el origen social para explicar las dificultades de aprendizaje en la consecución de las competencias. Asimismo presentamos la influencia que pueden 
ejercer en el desarrollo de las competencias básicas el tiempo dedicado al estudio fuera del centro educativo (Gráfico 9).

Dado que, al igual que en el apartado anterior, las tendencias se han revelado similares, sin que se describan diferencias, tanto en Educación Primaria como en ESO, los resultados se muestran con la distribución de las medidas de las variables ya analizadas anteriormente por su continuidad y porque, al menos, han sido evaluadas en tres cursos (Comunicación lingüística en castellano $(\mathrm{CB} \mathrm{CL}$ ), Competencia Matemática (CB MAT), Competencia en el conocimiento y la interacción con el mundo físico (CB CIMF) y Comunicación lingüística en inglés (CB ING).

El Índice Socio-económico y cultural (ISEC) muestra claramente una estrecha relación entre el nivel sociocultural del alumnado y el rendimiento mostrado en las evaluaciones diagnósticas de la cohorte, registrándose diferencias en todas las competencias y, de manera especial, en la Competencia lingüística en inglés. Otro dato a destacar son las tendencias registradas para cada competencia ya que muestran una gran estabilidad a pesar de los años, es decir, la procedencia del alumno parece bloquear de manera importante el rendimiento académico.

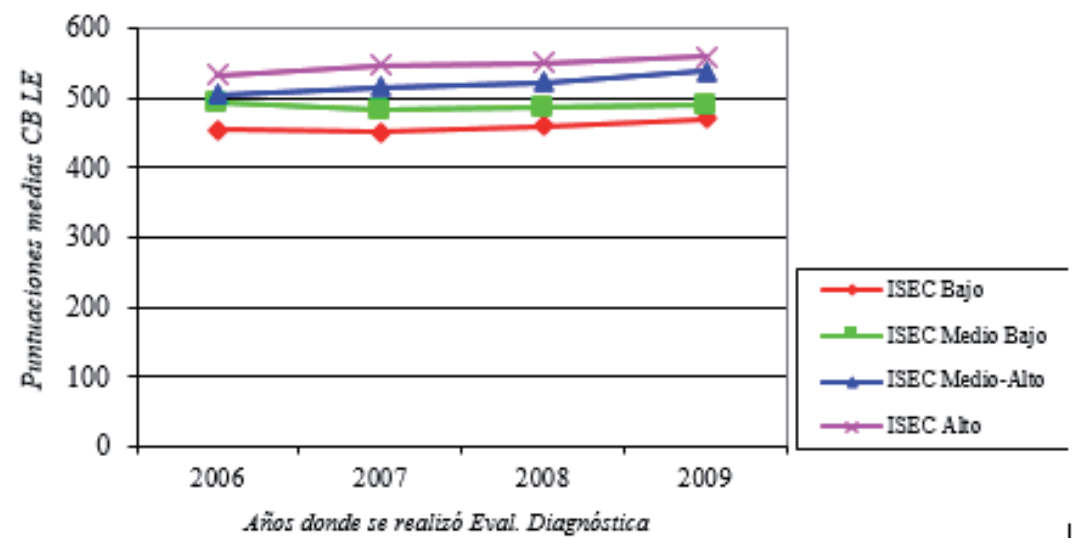

Fuente: Elaboración propia 
Enfoque por competencias y resultados escolares. Estudio longitudinal en el Principado de Asturias

José Antonio Álvarez Castrillón, Alejandro Rodríguez-Martín y Emilio Álvarez-Arregui
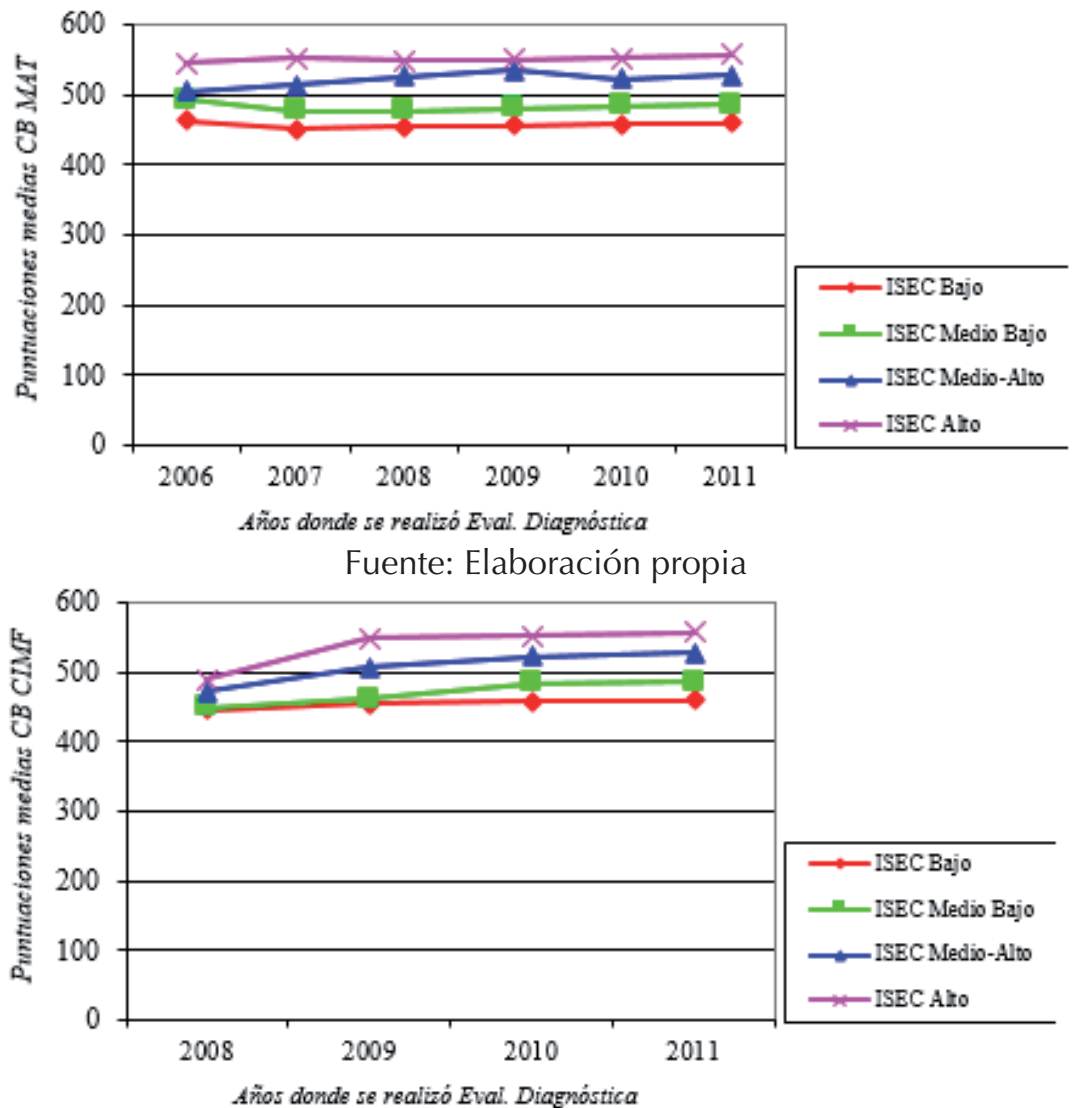

Fuente: Elaboración propia

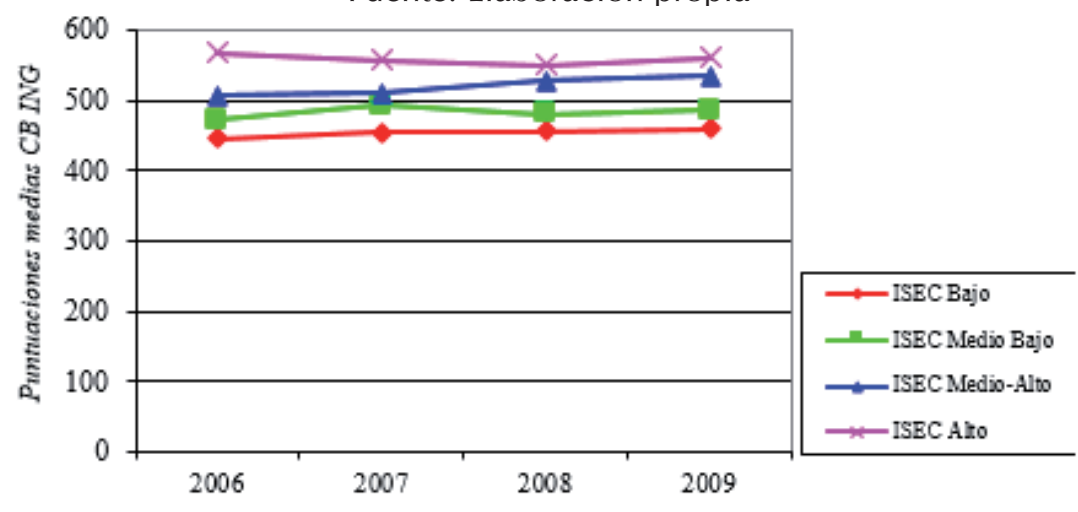

Años donde se realizó Eval. Diagnóstica

Fuente: Elaboración propia

Gráfico 8. Distribución de medias en relación con el Índice Socio-económico y Cultural (ISEC) y resultados por competencias 
La última de las variables analizadas en la cohorte es la el tiempo diario de estudio fuera del horario lectivo del centro educativo en el que está escolarizado el alumno. Los resultados obtenidos, similares en las áreas analizadas, nos permiten trazar una franja temporal óptima, entre 90-120 minutos, para la realización de tareas fuera del horario escolar. En los gráficos se observa que los resultados son más bajos cuanto se dedican entre 30-60 minutos y, aún más negativos, cuando el tiempo disminuya. Esta misma tendencia se produce cuando se sobrepasa esa franja ya que la escala de rendimiento comienza su declive.
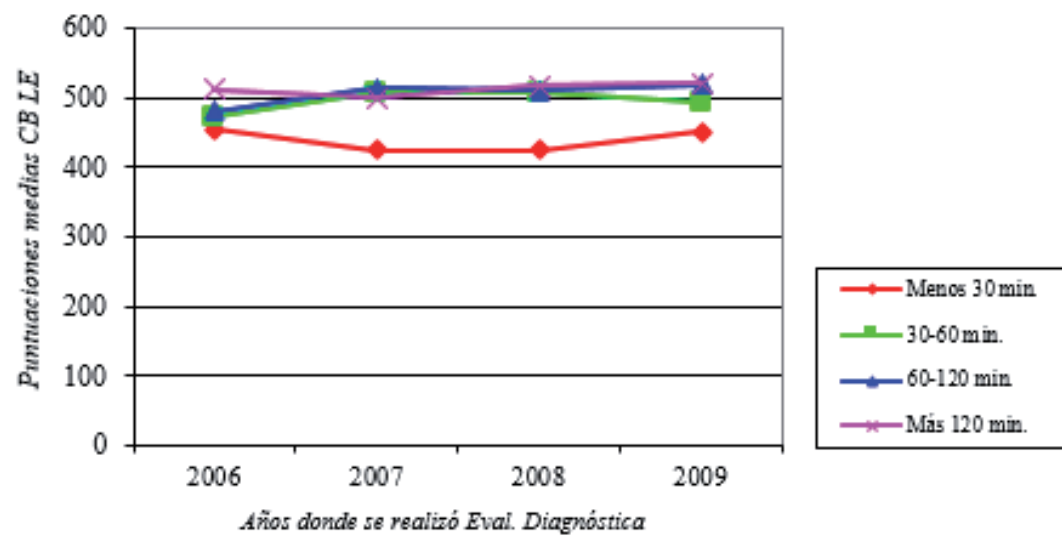

Fuente: Elaboración propia

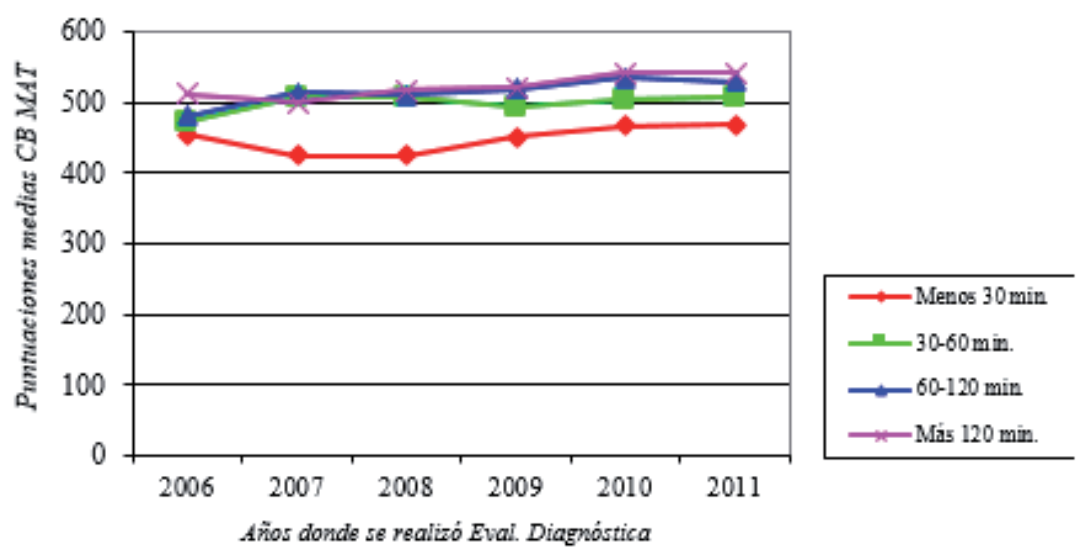

Fuente: Elaboración propia 
Enfoque por competencias y resultados escolares. Estudio longitudinal en el Principado de Asturias

José Antonio Álvarez Castrillón, Alejandro Rodríguez-Martín y Emilio Álvarez-Arregui

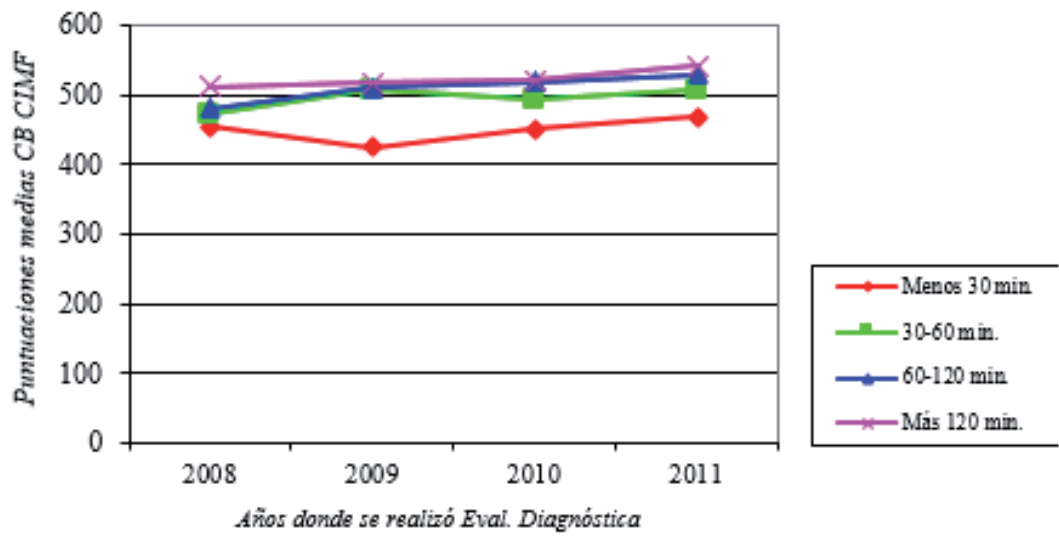

Fuente: Elaboración propia

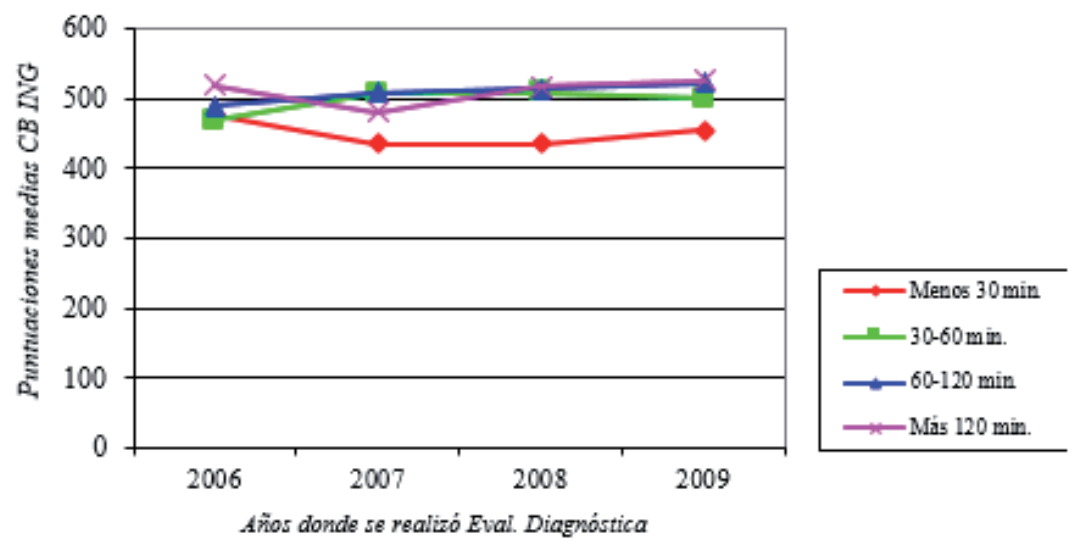

Fuente: Elaboración propia

Gráfico 9. Distribución de medias en relación con el tiempo dedicado al estudio fuera del centro educativo y resultados por competencias

\section{Conclusiones}

El meta-análisis realizado en la cohorte 2006-2011 nos ha permitido mostrar una visión global de la situación del Principado de Asturias respecto a los resultados obtenidos en las evaluaciones diagnósticas y el enfoque por competencias que se ha venido impulsando.

Atendiendo a los objetivos planteados en este estudio, destacamos las siguientes conclusiones: 
- Los niveles globales de rendimiento han ido mejorando a lo largo de los últimos en el Principado de Asturias y corroboran, además, la estabilidad del sistema educativo en esta Comunidad Autónoma.

- El alumnado asturiano ha ido incrementando su grado de adquisición de las competencias de manera progresiva en el período de tiempo analizado.

- Las características personales del alumnado son determinantes en la variación de los resultados. Esta cuestión, tal y como ha quedado reflejado en las últimas ediciones de PISA, avala la equidad del sistema. Las diferencias en el rendimiento responden más a factores individuales y al contexto socioeconómico del alumnado que a las variables organizativas y funcionales de los centros educativos.

- El sexo, el autoconcepto, repetir o no repetir curso y las expectativas académicas que se tengan están condicionando los resultados escolares del alumnado.

- Los centros educativos pueden afrontar, con garantías de éxito, iniciativas para trabajar el autoconcepto personal y académico (Trautwein, Luedtke, Kastens y Koeller, 2006),

- Los centros educativos pueden cambiar las expectativas y las motivaciones negativas que condicionan el rendimiento académico del alumnado cuando promueven la responsabilidad familiar en el aprendizaje (Dearing, Kreider y Weiss, 2008).

Además de las dificultades propias de la metodología desarrollada consideramos que el estudio aporta una visión de retrospectiva con proyección de futuro. Somos conscientes de que el estudio puede enriquecerse si se analizan todas las influencias que sobre el rendimiento académico y el logro de las competencias pueden ejercer el propio centro, su titularidad, el profesorado, sus años de experiencia y la metodología que emplea. Esta limitación constituye, en sí misma, una nueva oportunidad para seguir profundizando en este campo.

La propia complejidad de los Informes puede llegar a obstaculizar la necesaria difusión de su contenido en la comunidad educativa. En esta misma línea se aconseja que en futuras convocatorias se unifiquen las variables concurrentes así como la presentación de los resultados ya que con ello la comunidad científica podría realizar estudios comparativos con mayor eficacia. 
En este contexto nos parece relevante resaltar la necesidad de profundizar en la relación que existe entre las medidas de atención a la diversidad, el índice socioeconómico y cultural del alumnado y su rendimiento académico en las competencias evaluadas- a fin de conocer su impacto y si fuera necesario, impulsar el carácter inclusivo para el que fueron ideadas.

Los resultados obtenidos en evaluaciones de carácter institucional, como el caso que nos ocupa, ponen de relieve la importancia de conocer qué sucede en el sistema educativo con una vocación de mejora constante. La realidad educativa está cambiando y lo seguirá haciendo. La Ley Orgánica para la Mejora de la Calidad Educativa (LOMCE) nace, precisamente, para abordar un complejo escenario marcado por los resultados insuficientes de España en el Informe Pisa de 2009. Sin embargo nuestro sistema sigue manteniendo un grado de equidad importante. ¿Hasta cuándo? ¿Es posible seguir manteniéndolo? ¿Cómo conjugar realmente equidad y calidad?...Sería deseable que estos interrogantes se aborden claramente en la implementación de la LOMCE con evaluaciones periódicas y contextualizadas que sirvan, desde la corresponsabilidad de toda la comunidad educativa como mecanismo para la mejora y la participación.

\section{Referencias bibliográficas}

Álvarez, S., Pérez, A. y Suárez, M. L. (2008). Hacia un enfoque de la educación en competencias. Oviedo: Consejería de Educación y Ciencia.

Ananiadou, K. y Claro, M. (2009). 21st Century Skills and Competences for New Millennium Learners in OECD Countries. OECD Education Working Papers, $n^{\circ}$. 41. OECD Publishing. Recuperado: http://dx.doi.org/10.1787/218525261154

Barrero Fernández, B. y Domingo Segovia, J. (2010). Integrar competencias básicas y aprendizajes imprescindibles: Potencial curricular de las actividades globalizadoras. II Congreso Internacional de Didácticas. La actividad del docente: Intervención, Innovación, Investigación, Girona, Fecha 3-6 febrero. Recuperado de http://hdl.handle. net/10503/12297

Castro, M. (2010). ¿Qué sabemos de la medida de las competencias? Características y problemas psicométricos en la evaluación de competencias. Bordón, 63(1), 109123.

Cedefop (2008). Terminology of European education and training policy. A selection of 100 key terms. Luxembourg: Office for Official Publications of the European Communities. 
Enfoque por competencias y resultados escolares. Estudio longitudinal en el Principado de Asturias José Antonio Álvarez Castrillón, Alejandro Rodríguez-Martín y Emilio Álvarez-Arregui

Climént, J. B. (2010). Reflexiones sobre la Educación Basada en Competencias. Revista Complutense de Educación, 21(1), 91-106.

Collera, A. (2007). Evaluación de diagnóstico en Asturias: algunas notas de pretensión divulgativa. El Busgosu. Revista de investigación e innovación educativa, 6, 2-11.

Consejería de Educación y Ciencia del Principado de Asturias (2010). Evaluación de Diagnóstico. Asturias 2010. Oviedo: Consejería de Educación y Ciencia.

Dearing, E., Kreider, H. y Weiss, H. B. (2008). Increased family involvement in school predicts improved child-teacher relationships and feelings about school for low-income children. Marriage \& Family Review, 43(3), 226-254.

De la Orden, A. (2011). El problema de las competencias en la educación general. Bordón. Revista de Pedagogía, 63, 47-61.

Delors, J. (Coord.) (1996). La educación encierra un tesoro. Madrid: Santillana y Ediciones UNESCO.

Fernández-Alonso, R. y Muñiz, J. (2011). Diseño de cuadernillos para la evaluación de las Competencias Básicas. Aula abierta, 39(2), 3-34.

Jurado Valencia, F. (2009). El enfoque sobre competencias: Una perspectiva crítica para la educación. Revista Complutense de Educación, 20 (2), 343-354.

Lévy Leboyer, C. (2003). Gestión de las competencias. Barcelona: Ediciones Gestión 2000

Mulder, M., Weigel, T. y Collings, K. (2008). El concepto de competencia en el desarrollo de la educación y formación profesional en determinados países miembros de la U.E.: un análisis crítico. Profesorado. Revista de currículum y formación del profesorado, 12(3), 1-25.

Marín, F., Sánchez, J, Huedo, T. y Fernández, I. (2007). En A. Borges y P. Prieto (Eds.), Psicología y Ciencias afines en los albores del Siglo XXI (87-102). Granada: Grupo Editorial Universitario.

Monereo, C. (2003). Internet y competencias básicas. Aula de Innovación Educativa, 126, 16-20.

Monereo, C. y Pozo, J. (2007). Competencias para (con)vivir con el siglo XXI. Cuadernos de Pedagogía, 370, 12-17.

Morales, P. (1993). Líneas actuales de investigación en métodos cuantitativos: el metaanálisis o la síntesis integradora. Revista de Educación, 300, 191-221.

Murillo, F. J. (2007). Investigación Iberoamericana sobre Eficacia Escolar. Bogotá: Convenio Andrés Bello.

OCDE (2005). The definition and selection of key competencies. Executive summary. Recuperado de http://www.oecd.org/pisa/35070367.pdf

OCDE (2010). Working Paper 21st Century Skills and Competences for New Millennium Learners in OECD Countries (EDU Working paper $n^{\circ} .41$ ).

Oliveros Martín-Varés, L. (2006). Identificación de competencias: una estrategia para la formación en el Espacio Europeo de Educación Superior. Revista Complutense de Educación, 17 (1), 101-118.

Pérez Gómez, A. I. (2007). Reinventar la escuela, cambiar la mirada. Cuadernos de Pedagogía, 368, 66-71.

Perrenoud, Ph. (2004). Diez nuevas competencias para enseñar. Barcelona: Graó. 
Enfoque por competencias y resultados escolares. Estudio longitudinal en el Principado de Asturias

José Antonio Álvarez Castrillón, Alejandro Rodríguez-Martín y Emilio Álvarez-Arregui

Ribes, E. (2011). El concepto de competencia: su pertinencia en el desarrollo psicológico y la educación. Bordón. Revista de Pedagogía, 63, 33-45.

Roegiers, X. (2000). Saberes, capacidades y competencias en la escuela: una búsqueda de sentido. Aula de Innovación Educativa, 10, 103-119.

Roegiers, X. (2004). Une pédagogie de l'intégration. Bruxelles: DeBoeck Université.

Rosales López, C. (2010). La planificación de la enseñanza por competencias: ¿Qué tipo de innovación implica? Innovación educativa, 20, 77-88.

Rychen, D. S. y Hersch, S. L. (Eds.) (2003). Key Competencies for a Successful Life and a Well-Functioning Society. Cambridge, MA: Hogrefe \& Huber.

Sammons, P., Thomas, S. y Mortimore, P. (1997). Forging Links: Effective Schools and Effective Departments. London: Paul Chapman Publishing.

Sarramona, J. (2004). Las competencias básicas en la educación obligatoria. Barcelona: Ceac.

Sierra-Arizmendarrieta, B., Méndez-Giménez, A. y Mañana-Rodríguez, J. (2012). Necesidad y propuesta de un procedimiento para programar por Competencias Básicas. Aula abierta, vol. 40, no 3, 33-46.

Tiana, A. (2011). Análisis de las competencias básicas como núcleo curricular en la educación obligatoria española. Bordón. Revista de Pedagogía, 63, 63-75.

Trautwein, U. Luedtke, O, Kastens, C. y Koeller, O (2006). Effort on homework in grades 5 through 9. Development, motivational antecedents, and the associations with effort on classwork. Child Development, 77(4) 1094-1111.

Viso Alonso, J. R. (2010). Qué son las competencias. Vol.1. Madrid: EOS.

Zabala, A. y Arnau, L. (2007). 11 ideas clave. Cómo aprender y enseñar competencias. Barcelona: Graó. 